\title{
Mythos
}

Rivista di Storia delle Religioni

$13 \mid 2019$

Varia

\section{Il "maestro scomparso" nel rapporto epistolare tra Furio Jesi e Károly Kerényi}

The Role of the "Disappeared Master" in the Letters between Furio Jesi and Károly Kerényi

\section{Piero Rosso}

\section{(2) OpenEdition}

\section{Journals}

\section{Edizione digitale}

URL: http://journals.openedition.org/mythos/1506

ISSN: 2037-7746

\section{Editore}

Salvatore Sciascia Editore

\section{Notizia bibliografica digitale}

Piero Rosso, « II "maestro scomparso" nel rapporto epistolare tra Furio Jesi e Károly Kerényi », Mythos [Online], 13 | 2019, online dal 01 décembre 2019, consultato il 26 décembre 2019. URL : http:// journals.openedition.org/mythos/1506

Questo documento è stato generato automaticamente il 26 dicembre 2019

Mythos 


\title{
Il "maestro scomparso" nel rapporto epistolare tra Furio Jesi e Károly Kerényi
}

\author{
The Role of the "Disappeared Master" in the Letters between Furio Jesi and \\ Károly Kerényi
}

Piero Rosso

1 L'indole di Furio Jesi, più eterogenea che contraddittoria, nell'operare al confine tra materialismo ed esoterismo, alimenta ancora oggi il dibattito sul ruolo politico della mitologia. La morte prematura di Jesi nel 1980, il fatto che egli passò inosservato per un ventennio, offrono al lettore odierno una materia ancora malleabile, e la possibilità di entrare nell'officina di un pensatore che ha il potere di marcare il passo della critica. Le lettere di Jesi sono una finestra d'accesso, la "testimonianza che custodisce e tramanda la sua intuizione primigenia e nella quale è possibile leggere in trasparenza un vocabolario teorico che ritroviamo anche nelle opere più mature". ${ }^{1}$ Jesi infatti tornerà su questi testi con insistenza, ed essi troveranno una sistemazione compiuta nel suo Kierkegaard ${ }^{2}$.

Dalla metà degli anni ' 60 l'attività politica di Jesi si fa più intensa: ${ }^{3}$ cominciano le collaborazioni con le riviste "Quindici", "Uomini e idee" e "Resistenza. Giustizia e libertà", organo stampa dell'associazione "Giustizia e Libertà". All'interno di quest'ultima, Jesi fa parte dell'ala più a sinistra. ${ }^{4} \mathrm{Nel} 1968$ partecipa al dibattito aperto da Cottino, Levi e Dalmazzo sul numero 4 della rivista (aprile 1968) intorno alla "Guerra dei sei giorni" e al conflitto arabo-israeliano. In un articolo dal titolo Gli arabi e Israele e successivamente nel numero 8 di agosto dello stesso anno, con Israele, la democrazia e le grandi potenze - Jesi muove forti accuse a Israele, uno Stato "fondato da coloni di orientamento laico e nazionalista". ${ }^{5}$ Nato in una famiglia ebraica torinese, egli era toccato personalmente dalle sorti della "Guerra dei sei giorni", e in particolare dall'uso improprio del mito - l'affermazione di uno "Stato di diritto divino" nel nome del capitalismo imperialistico - sostenuto da alcuni suoi familiari. ${ }^{6}$ 
Già l'anno precedente aveva interpellato il maestro sulla questione nella lettera del 24 giugno 1967. La risposta di Kerényi, risalente al 30 luglio, è un punto di svolta importante nel rapporto tra i due, in quanto pone l'accento su delle questioni che si possono considerare alla base della rottura che avverrà nel maggio del 1968. Così Kerényi:

L'Ebreo è tornato ad essere, come in epoca nazista, la pietra di paragone: l'uomo è con lui, l'antiuomo contro di lui. Gli israeliani salvano l'immagine dell'uomo, e voler rendere inutile o del tutto nullo quest'atto di salvezza, attraverso calunnie, o speculazioni fuori dal mondo che senza volerlo sono parenti della calunnia - per non dire delle raffinate menzogne della propaganda -, è inumano. Non occorre che Le dica questo, se Lei si schiera con me dal punto di vista umanistico, che è insieme quello dello storico, dell'uomo normale e ragionevole! ${ }^{7}$

Kerényi era un sostenitore di un umanesimo fondato sul rapporto con il mito, che definiva una "guarigione"; non credeva che nel mito ci fosse spazio per un lato oscuro che corrompesse l'uomo, bensì che l'uomo corrompesse il mito ${ }^{8}$. Su questo era in disaccordo con Thomas Mann, che da giovane aveva a sua volta considerato un maestro. Mann, infatti, guardava al mito con molta diffidenza, lo considerava "potenzialmente umano" e "potenzialmente demoniaco". Il Doktor Faustus, che esce poco dopo la seconda guerra mondiale, è il punto estremo di questa inquietudine che lo scrittore sente agitarsi nell'inconscio; è il romanzo del proprio senso di colpa di fronte al nazismo, in quanto uomo e in quanto tedesco, su cui preme lo spettro di Ernst Bertram, l'amico "nazista aristocratico" sedotto dal regime'. La parole di Kerényi nella lettera del 30 luglio 1967 anticipavano le critiche rivolte a Jesi in seguito alla pubblicazione de Gli arabi e Israele - la formula "raffinate menzogne della propaganda" ritorna quando Sion Segre-Amar definisce l'articolo "sottili menzogne e malevole fantasie"10 - ed erano sintomo di una divergenza intellettuale profonda sull'essenza dell'umanesimo. L'Ebreo diventava pertanto "pietra di paragone" che separava l'umanesimo dalla calunnia. Per Kerényi, che non accettava il demonismo del mito paventato da Mann, questa distinzione era fondamentale. In realtà, l'incompatibilità di posizioni intorno all'umanesimo tra Jesi e Kerényi era già stata esplorata dal primo nel celebre articolo Cesare Pavese, il mito e la scienza del mito ${ }^{11}$ del 1964, di cui Kerényi ignorava l'esistenza. In esso Jesi rimproverava al maestro una "mascheratura umanistica" e cominciava a esprimere disagio verso la possibilità di una dedemonizzazione del mito. ${ }^{12}$ Le lettere di Kerényi del 25 giugno 1965 e del 30 luglio 1967 permettono di comprendere meglio l'essenza del suo umanesimo: nella prima egli chiarisce, a proposito del nazismo, di non avere una spiegazione mitologica bensì "rigorosamente scientifica"13; nella seconda evoca un "punto di vista umanistico, che è insieme quello dello storico, dell'uomo normale e ragionevole" ${ }^{14}$. È un appello alla razionalità che Jesi non condivide, poiché egli si trova piuttosto in una "posizione intermedia tra credenza e scienza, fra razionalità e interiorità" ${ }^{15}$.

5 In primo luogo, Jesi era lettore di Aby Warburg, le cui le categorie di analisi, secondo Agamben, erano "infinitamente più sottili della contrapposizione fra razionalismo $\mathrm{e}$ irrazionalismo" e secondo il quale il conflitto andava interpretato "in termini di polarità e non di dicotomia". ${ }^{16}$ Partendo da Kerényi e da Jung, Jesi aveva lavorato fin da subito per evitare la definizione del significato del mito, preferendo tracciare gli spostamenti e le trasformazioni di ciò che chiamava "materiali mitologici":

Sin dai suoi esordi Jesi si è concentrato non sul significato dei materiali, ma sulle loro "affinità elettive", spostando l'accento dalle "immagini" della teoria junghiana classica, alle "connessioni" - le sole che egli intende come "archetipiche" - capaci 
di legare immagini e concetti al di là dei rapporti logici [...] il patrimonio della mitologia si presenta cioè allo sguardo non come una riserva di immagini dense di significato, ma come un campo di pure relazioni tra figure di per sé non significanti ${ }^{17}$.

Sulla base di questo metodo, acentrico come l'atlante Mnemosyne di Warburg, egli aveva rielaborato l'umanesimo di Kerényi in un'antropologia del frammento, o meglio nel “più avanzato tentativo in Italia di porre le basi per un'antropologia dell'immagine, e dell'immagine mitologica in particolare, nonché la più lucida teorizzazione, dopo Warburg [...] del tema delle 'sopravvivenze' visuali del mito"18. Questa posizione intermedia tra razionalismo e irrazionalismo, ricorda Manera, ha dato luogo a fraintendimenti. Calvino descrisse Jesi "uno Zolla che gira a rovescio, a sinistra", interessante nel suo "volare con la scopa":

A noi che abbiamo imparato ad apprezzare in Jesi il ricercatore che sonda gli sfondi irrazionali della razionalità con strumenti iper-razionali e che stana i tanti volti della 'cultura di destra', colpisce il disagio che la sinistra intellettuale provava per chi studiava l'irrazionalismo' - nome di comodo per qualcosa di molto più complesso ${ }^{19}$.

7 In secondo luogo, sia Warburg che Jesi apportano "contributi a scienze ancora da fondare" ${ }^{20}$ : il torinese riconosce l'inadeguatezza della scienza del mito, che rappresenta quest'ultimo come un'oggettivazione e non come una polarità, e ne ricerca il superamento in maniera più sistematica a partire dagli anni settanta ${ }^{21}$.

Rileggendo ora la lettera di Kerényi del 30 luglio 1967 ci troviamo di fronte a una forma di umanesimo che per negare qualunque corruzione del mito che non sia di matrice umana divide ancora dicotomicamente ciò che è umano da ciò che non lo è. Le lettere su Israele si trovano dunque a un crocevia importante nel percorso intellettuale di Jesi, se consideriamo che il 1967 è anche l'anno di pubblicazione di Germania segreta, opera che Jesi termina nonostante l'opinione sfavorevole del maestro; che il 1968 è l'anno di Letteratura e mito e della conseguente rottura con Kerényi e che il 1969 è dedicato al lavoro su Spartakus, pubblicato solo postumo, nel quale Jesi cerca di superare l'umanesimo kerényiano. Questi tre testi dialogano con il corpus dell'epistolario JesiKerényi e permettono di comprendere come il loro rapporto, di natura pedagogica, dipenda dallo sviluppo delle teorie sul mito.

La formula Germania segreta (Das geheime Deutschland) aveva avuto molta risonanza nella cultura tedesca a partire dal primo dopoguerra, per indicare una comunione dei grandi spiriti della nazione, una Germania buona, un Olimpo tedesco con cui il poeta Stefan George cercava il contatto tramite il raccoglimento intorno al proprio circolo letterario. La stessa formula era stata usata dallo storico Erns Kantorowicz come titolo della sua prima lezione, dopo l'autosospensione dall'insegnamento per protesta contro il nazismo ${ }^{22}$. Jesi procede in direzione opposta, come testimonia la lettera del 16 maggio 1965:

Se analizzo il più freddamente possibile la mia posizione, vi ritrovo una sorta di fatalismo (nel quale riconosco la mia eredità ebraica) dinanzi alle colpe di cui l'uomo è capace, unito ad una forma di "comprensione" nei confronti dei colpevoli: comprensione che - lo so - potrebbe essere considerata colpevole essa stessa, come complicità [...]. Ma credo di riconoscere nell'opera di Hitler qualcosa che trascende le responsabilità umane; credo insomma che il vero colpevole degli orrori del nazismo non sia stato l'uomo-Hitler, ma una forza temibile quanto gli Angeli di Rilke che si è servita di quell'uomo, invadendo la sua volontà̀ ${ }^{23}$. 
Germania segreta per Jesi è la formula che punta il dito contro "le forze oscure agenti nella tragedia tedesca" ${ }^{24}$, per monitorare l'epifania del mito e tracciarne le metamorfosi, anche a rischio di subirne l'influenza:

Penso di essere cosciente dei pericoli insiti nell'accostare intimamente - come mi propongo di fare - una vicenda di orrore e di morte: il contagio di un tal genere di male trova sempre facile terreno dentro di noi ${ }^{25}$.

11 A questo primo tentativo fa seguire Spartakus, che dedica a Rosa Luxemburg, guida della rivolta spartachista del 1919 assieme a Karl Liebknecht. L'esperienza berlinese era stata un esempio di applicazione pratica delle teorie marxiane, e aveva scatenato un dibattito sull'effettiva adesione di queste realtà all'ortodossia. L'eco delle vicende, giunto in Italia ${ }^{26}$, genera interpretazioni molto differenti su cui Jesi ritiene doveroso fare chiarezza. Negli anni sessanta, nel generale clima di rilettura degli eventi politici, il caso tedesco gli si presenta come l'occasione di liberare la figura della filosofa dalla narrazione che ne dipinge la morte come un errore di valutazione o, ancora peggio, un "malinteso sentimento dell'onore" ${ }^{27}$. Nelle pagine di Spartakus, partendo dalle posizioni critiche di Rosenberg, Adler, Zetkin, Jesi imposta un ragionamento in senso opposto, che segue l'affermazione di György Lukács in Storia e coscienza di classe sull'unità di teoria e prassi nelle idee di Luxemburg ${ }^{28}$. Rifiuta inoltre di seguire il sentiero della monografia storica per cercare piuttosto di "avvicinarsi alla sua vicenda spirituale ${ }^{29}$.

Se l'unità di teoria e azione, in cui Luxemburg crede fermamente, prevede la sconfitta nel quadro più ampio della lotta di classe, la mancata fuga dei capi del movimento è da imputarsi all'involontaria accettazione degli stessi simboli contro cui essi sono chiamati a combattere. Nel novembre 1918 Luxemburg scrive: "tutti noi siamo soggetti alla cieca sorte, e mi conforta soltanto il pensiero che forse anch'io presto sarò spedita nell'aldilà - forse da una pallottola della controrivoluzione che da tutti i lati è in agguato" ${ }^{30}$. Jesi evidenzia la polarizzazione del pensiero che avviene nel tempo della rivolta e che si muove su un asse che contrappone la vittoria alla morte ${ }^{31}$. Le posizioni di Luxemburg, all'apparenza così lontane dall'umanesimo borghese di Kerényi, sembrano a tratti accostarvisi nella considerazione di un tempo lineare in cui il passato (Kerényi) e il futuro (Luxemburg) coincidono con il tempo della "guarigione", a discapito di un presente troppo umano:

Non va dimenticato, infine, che proprio la superiore visione etico-politica della Luxemburg, in base alla quale essa non poteva dissociare completamente la rivolta dalla rivoluzione, la rendeva più suscettibile della folgorazione di conoscenza implicita nella rivolta, e cioè le poneva dinanzi in modo fascinatorio - proprio a lei, così acuta indagatrice della struttura economica del capitalismo - l'avversario come il nemico demonico ${ }^{32}$.

13 Il confronto con Kerényi appare significativo proprio di fronte a questo rimando al demonismo. Perché ammettendo l'esistenza e la successiva scomparsa nell'orizzonte moderno del "mito genuino" 33 - più alto di ogni male e di ogni bene, luogo e tempo "ove il dèmone non può esistere" ${ }^{34}$ - egli individua nel presente una dimensione "non genuina" dell'esistenza. Rosa Luxemburg demonizzando l'avversario, ponendo la lotta sullo stesso piano dell'umano e dell'anti-umano che Kerényi interpella nella lettera del 30 luglio 1967, "si costituisce come normale, ossia [...] conserva e garantisce suscitando un'alterità a cui conferire un volto demonico, estraneo alla vita e alla storia" ${ }^{35}$. Jesi riconduce questa adesione simbolica a un effetto del discorso capitalista. È interessante ricordare che, da sindacalista, egli offre anche uno sguardo dall'interno sul fenomeno per cui "le organizzazioni della classe operaia da molto tempo hanno preso a modello le 
strutture avversarie", poiché il discorso capitalista è riuscito a infondere un valore simbolico di potere a tali strutture ${ }^{36}$. In questo senso gli spartachisti avrebbero fatto il gioco del nemico:

La micidiale forza fascinatoria dei simboli del potere capitalistico perdura anche quando non si tratta neppure più di conquistare quei simboli; resta, infatti, la certezza che quei simboli siano in qualche modo - pur orrido e colpevole - un "vertice", un'epifania di potenza, e che quindi si debba contrapporre loro un'epifania di virtù per acquistare la medesima potenza ${ }^{37}$.

Fino a pochi anni prima questo potere fascinatorio coinvolgeva lo stesso Jesi nel suo rapporto epistolare con Kerényi, come si vede in particolare nelle missive del 1965.

Anche nel torinese avviene, a metà degli anni sessanta, una parziale e temporanea accettazione dei simboli del potere, poi definitivamente rifiutata alla fine di quel decennio con la scrittura di Spartakus. Si tratta della discorsività del maestro, dell'influenza esercitata sull'allievo da una figura che per Jesi, fino all'incontro tra i due il 27 e 28 maggio 1965 al Museo Egizio di Torino, era sempre rimasta nel dominio della letteratura. Ne testimonia la lettera del 30 maggio 1965, scritta dunque due giorni dopo la visita torinese di Kerényi, in cui Jesi ripete parola per parola le formule che gli erano state suggerite dal maestro nelle lettere precedenti e in cui sostiene di aver esperito una vera "guarigione dai demoni". Nella lettera del 16 gennaio 1965 Jesi si dichiara dubbioso sulla possibilità di de-demonizzare il mito ${ }^{38}$; il 15 maggio esprime ancora dubbi sulla a proposito di una "guarigione":

La mia situazione attuale assomiglia a quella dei personaggi del Demian di Hesse: un atteggiamento di fronte al divino o all'extra-umano che non esclude in esso la parte più terrifica, di orrore e di distruzione. Per questo ebbi a scriverLe in passato che ponevo qualche dubbio sulla possibilità e l'efficacia di una 'guarigione' dell'uomo ${ }^{39}$.

Il 30 maggio, invece, accetta la versione dell'umanesimo kerényiano:

Nell'istante in cui mi sentivo più debole a reagire contro il mito "non genuino", deformato attraverso la lente del demonismo, ero in realtà più vicino all'inizio della "guarigione": all'istante in cui il dèmone non mi sarebbe più apparso come parte integrante del dio ${ }^{40}$.

E ancora:

Il mito - il mito genuino - non è l'essenza del bene o del male affrontati e congiunti, ma "un punto più in alto", ove il demone non può esistere, giacché il demone può essere solo dentro di noi, come un tragico "errore di vista" o come una forza da vincere, giacente nel nostro sguardo: non sull'orizzonte della realtà ${ }^{41}$.

Jesi passa così dal citare il Demian di Herman Hesse, in cui è ben presente l'oscillazione tra bene e male, al dividere l'influsso benefico del "mito genuino" da quello umano, senza esitazioni o soluzioni intermedie. Nell'arco temporale coperto dalle lettere del 1965, l'ambivalenza che Jesi sente di dover affrontare e mantenere nei confronti del mito viene messa da parte. Alla luce di questo cambio di rotta si potrebbe approfondire l'affermazione di Gnoli, fatta in occasione della pubblicazione del carteggio nel 1999, su come nelle prime lettere di Jesi vi sia una "sottomissione" nei confronti di Kerényi, un atto particolarmente significativo se compiuto da un giovane che aveva fin da subito rivendicato il proprio ruolo di allievo irregolare ${ }^{42}$. Prosegue Gnoli: "è come se, rivolgendosi a Kerényi, gli dicesse che il solo possibile luogo di incontro tra il maestro e l'allievo è nella profondità dell'animo" ${ }^{43}$. Jesi, con la scrittura di Cesare Pavese, il mito e la scienza del mito, aveva già dichiarato la sua irregolarità rispetto a tale scienza, una posizione che ribadirà nel 1967 con Germania segreta, nel 1969 con Spartakus e che poi proseguirà in una sistemazione più metodica nei saggi che compaiono negli anni settanta, a partire da Mito. L'incontro spirituale tra i due sembra realizzarsi solamente 
nella lettera del 30 maggio ed esclusivamente nell'atto di sottomissione dell'allievo al discorso del maestro, nell'eliminazione temporanea di quella scomoda inquietudine che interroga le polarità del mito e che da lì a poco, con l'avvicinamento al materialismo di Walter Benjamin da parte del giovane autore, non esiteremmo a definire "dialettica". Si potrebbe infatti ipotizzare che la malattia da cui Jesi in più occasioni si sente affetto coincida con la lenta e progressiva acquisizione di uno sguardo dialettico sulla storia.

L'appiattimento dello sguardo di Jesi sull'orizzonte di Kerényi non dura a lungo, poiché il momento di massimo avvicinamento tra i due mitologi segna anche l'inizio della crisi del loro rapporto. Nel momento in cui Jesi accetta le parole del maestro e le sostituisce alle proprie crea la necessità di scardinarle, perché la nozione di mito genuino "esige per Jesi, e contrariamente a Kerényi, la possibilità, in quell'ordine imprevedibile, del suo totale sovvertimento" ${ }^{44}$. Oltre al caso della lettera del 30 maggio 1965, anche le teorie esposte nel lavoro che Jesi compone su Kierkegaard sono testimoni di quest'attitudine ${ }^{45}$.

Manera ricorda che il saggio di Kerényi, Origine e fondazione della mitologia, posto ad introduzione dei Prolegomeni di Jung e Kerényi, sviluppa in Jesi l'idea che la mitologia sia movimento e rielaborazione di "mitologemi" ${ }^{46}$. Se è vero che "anche il potere è comunicabile" ${ }^{47}$ si capisce perché la critica di Jesi, negli anni della militanza politica torinese, si concentri sui movimenti di materiali culturali. Egli ritrova in Kierkegaard molte occasioni di sovvertimento perché al sapere oggettuale e al nozionismo potremmo dire: alla trasmissione di un potere come nozionismo - egli contrappone la trasmissione dell'etica. L'etica è per Jesi un bene universale e immediato che richiede solamente una realizzazione ${ }^{48}$; nella comunicazione efficace dell'etica, il maestro è chiamato a incarnare l'insegnamento, dato che l'etica stessa non può essere reificata. Essa dev'essere veicolata con la scelta attiva di un diverso modo di comunicare. Dato che il maestro non può definire e spiegare l'etica come un oggetto, la comunicazione che la riguarda è sempre un atto indiretto. La capacità del filosofo danese di distanziarsi dalla propria voce, di uscire dal proprio linguaggio attraverso l'uso dell'ironia si rispecchia nel frequente utilizzo di pseudonimi:

Lo pseudonimo è il dispositivo con cui Kierkegaard evita l'espressione diretta e annulla ogni volta il contenuto del sapere, comunicando invece, indirettamente, qualcosa di essenziale sulla propria attività di scrittore, rendendola cioè eticamente vera $^{49}$.

È in base a questo rifiuto che si può trovare nell'intera opera di Kierkegaard un'autobiografia nascosta ${ }^{50}$ così come succede per Jesi: l'autore "non ha esitato ad accostare la sua personale esperienza del vissuto e la sua capacità di riflessione critica ai punti più problematici della riflessione, dove precisione storica e interpretazione della metafora mitica s'incontrano" ${ }^{11}$. Nell'operare con materiali storici o filosofici, Jesi non è più un mero osservatore in balia di forze mistiche. "Alla fissità del ritratto, Jesi sostituisce il tentativo di inserirsi nel pieno di un movimento o campo di forze maieutico" e parallelamente non vuole ridurre Kierkegaard - e Rosa Luxemburg, nel caso di Spartakus - a "soggetto di una monografia", ma coglierlo "nel corso della sua stessa scrittura" ${ }^{52}$. Allo stesso modo l'eredità di Kerényi, che nella lettera del 30 maggio 1965 è vissuta da Jesi ancora in maniera nozionistica - si consideri il tono recitativo, quasi forzato, della lettera in questione - all'inizio degli anni '70 assume già i tratti sovversivi di un"'eredità indiretta":

Ecco la formula di un pensiero-movimento e di un soggetto - irriducibile alle identità del maestro o dell'allievo, del lettore o dello scrittore [...]. Maestro e allievo 
non saranno mai come l'originale e la sua replica, poiché sono già inseparabili, come il tempo ed il pensiero ${ }^{53}$. l'arco del suo apprendistato epistolare con Kerényi, e costituisce una riflessione finale su quelle esperienze. Il rapporto che Jesi instaura con il maestro nel Kierkegaard, non è lo stesso che si costruisce lettera dopo lettera con Kerényi. Si tratta piuttosto dell'elaborazione di quel rapporto come un insieme di polarità e non come una dicotomia. Il rispetto di questo punto, è particolarmente importante per la critica jesiana perché la trappola mitologica di considerare la frattura tra Jesi e Kerényi come l'origine (Ur) dello Jesi maturo è sempre in agguato ${ }^{54}$. Il maestro e l'allievo che si delineano nel Kierkegaard sono dunque molto diversi dalle figure storicamente determinate dello Jesi-allievo e del Kerényi-maestro nei primi anni del loro scambio epistolare. Se nella lettera del 30 maggio 1965 Jesi giunge a recitare al maestro proprio le sue stesse parole, significa che in quell'attimo egli accetta di diventare

Una precisa replica del maestro: ovvero colui che, subendone l'influenza, sia riuscito anche a carpirne la tattica, a segnarne con precisione i tratti e possederli talmente da poterli ripetere, facendosi maestro lui stesso - ma anche trasformando la didattica in scienza, il potere in oggettività, fallendo insieme come maestro e come allievo ${ }^{55}$.

Senza le interruzioni e le ambiguità nel discorso di chi insegna, l'insegnamento stesso, quale era per Jesi l'umanesimo kerényiano, sfocia nella conservazione. Nel frammento inedito Rivelazione e morale, ritrovato da Cavalletti tra le carte di Jesi, si trova una riflessione a proposito di Rilke: "l'opera pedagogica compiuta da Rilke verso i suoi corrispondenti fu sempre, esclusivamente, propedeutica. Egli guidò i passi di chi gli si rivolgeva fino alle soglie della verità. Poi si ritrasse, e lasciò che ciascuno in solitudine proseguisse il suo cammino" ${ }^{56}$. Il vero maestro allora deve scomparire dalla "visuale dell'allievo" per far sì che si realizzi il "vero momento maieutico" ${ }^{57}$. Che Jesi abbia accolto questa idea con il passare degli anni, è confermato da Mito, pubblicato nel 1973 all'interno dell'Enciclopedia Filosofica dell'ISEDI di Milano, quasi contemporaneo al Kierkegaard $^{58}$. In esso, forse mai come prima, lo stesso Jesi si pone nei panni del maestro e pedagogo che discorre criticamente sulla scienza del mito. Nell'introduzione a questo testo, però, Jesi fornisce una rilettura della morte di Socrate: la prima parola di Socrate in Mito è pertanto anche l'ultima. Possiamo leggere in questa scelta compositiva una dichiarazione di intenti su quale tipo di maestro Jesi volesse incarnare, quando l'ombra di Kerényi si era già fatta decisamente più sottile. È certo che il Kerényi in praesentia al Museo Egizio di Torino si avvicinò, per Jesi, alla figura del vero maieuta; ma il rapporto unicamente epistolare che precedette e che seguì l'incontro tra i due facilitò la rielaborazione della figura del Kerényi-maestro nelle opere che seguirono.

Le lettere del 1965 e del 1967 fanno trasparire con particolare chiarezza il tentativo del maestro ungherese di affermare il proprio discorso a discapito di quello dell'allievo, dimensione che non si sarebbe mai potuta adattare alla personalità poliedrica $\mathrm{e}$ irregolare di Jesi, che è obbligato perlopiù a trattenere i suoi dubbi e le sue inquietudini nei confronti del mito "genuino" e dell'umanesimo kerényiano. Le parole, infatti, se non uscirono dalle lettere di Jesi, raggiunsero la scrivania di Kerényi nella forma del saggio Cesare Pavese, il mito e la scienza del mito, appena ripubblicato in Letteratura e mito. Kerényi si sentì punto al cuore da Jesi sulla questione dell'umanesimo che molti anni prima aveva dibattuto con Mann. Kerényi considerava Mann suo maestro, per cui la possibilità di "guarigione" data dal mito "genuino" - in cui Mann non credeva, al punto

Mythos, 13 | 2019 
di scrivere un romanzo di auto-condanna - non rappresentava solamente un punto chiave della teoria kerényiana, ma anche uno dei momenti grazie ai quali Kerényi poté finalmente spogliarsi degli abiti dell'allievo. La risposta di Kerényi al saggio di Jesi, infatti, rientra a pieno titolo nella parola del maestro che non vuole ritirarsi come Rilke:

Ora però vi ho letto per la prima volta il Suo lavoro Cesare Pavese, il mito e la scienza del mito. Lei non me lo inviò quando apparve in "Sigma", poiché forse non era proprio sicuro che io potessi trarne diletto e dare il mio consenso. Meglio sarebbe stato, se una tale inquietudine - ammesso che non si tratti d'una mia pura supposizione - fosse persistita, continuando fino a oggi ${ }^{59}$.

La sua visione dicotomica che Kerényi instaura nei suoi rapporti maestro-allievo comporta che ogni frattura (Ur-) diventi un abisso. Jesi, con il concetto di "mascheratura umanistica", aveva denudato Kerényi dell'abito di maestro. La risposta dell'ungherese, nell'ultima lettera del 1968, contiene una critica forte e personale all'attività "italo-comunista" di Jesi, che fin dalle lettere del 1967, quando prende parte al dibattito su Israele, era risultata un motivo di forte divergenza. Se le considerazioni sulla pedagogia che Jesi trae dalla scrittura del Kierkegaard sono valide, significa che la frattura Kerényi-Jesi da parte di Kerényi non ebbe lo stesso valore della ripolarizzazione Jesi-Kerényi da parte di Jesi. Quest'ultimo, in altre parole, fu capace di rielaborare la figura del maestro in altre forme. Delle parole di Kerényi, sulle quali Jesi non smise mai di tornare, rimane una traccia forte nel Kierkegaard e nelle scritture successive alla sua morte, avvenuta il 14 aprile 1973. Nel discorso funebre tenuto per il maestro ad Ascona l'8 novembre 1974, Jesi rifiuta il ritratto monografico, "affinché il ricordo sia critica e vita anziché agiografia e morte" ${ }^{00}$ per "ricordare sì, ma in modo creativo" ${ }^{61}$, a dimostrazione del fatto che la scomparsa del maestro non ebbe nulla a che fare con la sua morte.

\section{BIBLIOGRAFIA}

AGAMBEN 1984: G. Agamben, «Aby Warburg e la scienza senza nome», Aut aut. Rivista di filosofia e di cultura, 199-200 (1984), 51-66.

BELLISARIO 1942: V. Bellisario, «Il dramma di Kierkegaard», Rivista di Filosofia Neo-Scolastica 34-3 (1942), 127-136.

BELPOLITI, MANERA 2010: M. Belpoliti, E. Manera (a cura di), Furio Jesi, Riga 31, Milano 2010.

CAVAlLETti 1999: A. Cavalletti, Demone e immagine. Carteggio 1964-1968, in F. Jesi, K. Kerényi, Demone e mito, Macerata 1999, 127-151.

CAVALLETTI 2000: A. Cavalletti, «Leggere Spartakus», in F. Jesi, Spartakus. Simbologia della rivolta, Torino 2000, VII-XXVIII.

CAVAlLETti 2001: A. Cavalletti, «Postfazione. Il metodo della scrittura indiretta», in F. Jesi, Kierkegaard, Torino 2001, 203-223. 
CAVAlLETTI 2001: A. Cavalletti, «La maniera compositiva di Furio Jesi», in F. Jesi, Materiali mitologici. Mito e antropologia nella cultura europea, Torino 2001, 359-376.

COLLOTTI 1969: E. Collotti, «I socialisti italiani e la rivoluzione di novembre in Germania», Studi Storici 10, 3 (1969), 587-611.

COMETA 2010: M. Cometa, L'immagine in Jesi, in M. Belpoliti, E. Manera (a cura di), Furio Jesi, Riga 31, Milano 2010.

FERRARI 2010: R. Ferrari, «Il maestro e l'allievo», in M. Belpoliti, E. Manera (a cura di), Furio Jesi, Riga 31, Milano 2010, 166-181.

GIACHETTI 2011: D. Giachetti, Per la giustizia e la libertà. La stampa Gielle nel secondo dopoguerra, Milano 2011.

GNOLI 2010: A. Gnoli, «Travolti da un insolito mito», in M. Belpoliti, E. Manera (a cura di), Furio Jesi, Riga 31, Milano 2010, 52-54.

JESI 1968: F. Jesi, «Cesare Pavese, il mito e la scienza del mito», Sigma 3-4 (1964), 95-120, ora in Letteratura e mito, Torino 1968, 129-160.

JESI 1968: F. Jesi, «Gli arabi e Israele. Sionismo politico e spirituale», Resistenza. Giustizia e Libertà 22, 6 (1968), 2, ora in M. Belpoliti, E. Manera (a cura di), Furio Jesi, Riga 31, Milano, 2010, 56-65. JESI 1995: F. Jesi, Germania segreta. Miti nella cultura tedesca del ‘900, Milano, 1995.

JESI 2000: F. Jesi, Spartakus. Simbologia della rivolta, Torino, 2000.

JESI 2001: F. Jesi, «Károly Kerényi. L'esperienza dell'isola», in F. Jesi, Materiali mitologici. Mito e antropologia nella cultura europea, Torino 2001, 54-66.

JESI 2013: F. Jesi, «Quando Kerényi mi distrasse da Jung», in F. Jesi, Il tempo della festa, Roma 2013, 224-231.

JESI, KERÉNYI 1999: F. Jesi, K. Kerényi, Demone e mito. Carteggio 1964-1968, Macerata 1999.

JUNG, KERÉNYI 1972: C. G. Jung, K. Kerényi, Prolegomeni allo studio scientifico della mitologia (ed. or. Amsterdam 1941), trad. it., Torino 1972.

KERÉNYI 1964: K. Kerényi, «Dal mito genuino al mito tecnicizzato», in E. Castelli (a cura di), Tecnica e casistica: tecnica, escatologia e casistica (Colloquio di Roma, 7-12 gennaio 1964), Roma 1964, 153-168.

LUKÁcs 1973: G. Lukács, Storia e coscienza di classe (ed. or. Berlin, 1923), trad. it., Milano 1973. MANERA 2012: E. Manera, Furio Jesi. Mito, violenza, memoria, Roma 2012.

MANERA 2015: E. MANERA, «Furio Jesi scrittore. A proposito de L'ultima notte», Doppiozero, 2015, consultato il 30 settembre 2019, URL: doppiozero.com/materiali/recensioni/furio-jesi-scrittoreproposito-de-lultima-notte

SANTINI 2013: C. Santini, «La seduzione del mito. Furio Jesi, Thomas Mann e Karoly Kerényi», in S. Fornaro, D. Summa (a cura di), Eidolon. Saggi sulla tradizione classica, Bari 2013, 107-124.

SCHIAVONI 2010: G. Schiavoni, «L'uomo segreto che è in noi», AA.VV, Immediati dintorni. Un anno di psicologia analitica, Milano 1989, ora in M. Belpoliti, E. Manera (a cura di), Furio Jesi, Riga 31, Milano 2010, 271-278. 


\section{NOTE}

1. COMETA 2010, 260.

2. CAVAlLETTI 1999, 131.

3. BELPOLITI, MANERA 2010, 13-17.

4. GIACCHETTI 2011, 156.

5. IVI, 112.

6. JESI, KERÉNYI 1999, 106-108.

7. Ivi, 104-105.

8. Cfr. JESI, KERÉNYI 1999, 57-59: attribuire al mito gli orrori perpetrati è una "scappatoia meschina".

9. SANTINI 2013, 113.

10. GIACCHETTI $2011,112$.

11. JESI 1968, 129-160.

12. CAVALLETTI 1999, 135.

13. JESI, KERÉNYI 1999, 57.

14. Ivi, 104-105.

15. FERRARI 2010, 4-5.

16. AGAMBEN 61, n. 30.

17. CAVALLETTI 2001, 363.

18. COMETA 259.

19. MANERA 2015.

20. AGAMBEN 1984, 54, n. 12.

21. SCHIAVONI 2010, 271.

22. SANTINI 2013, 119. Si veda anche l'articolo di Carlotta Santini in questo numero.

23. JESI, KERÉNYI 1999, 51.

24. Ivi, 50 .

25. Ibidem.

26. COLLOTTI 1969, 609.

27. JESI $2000,15$.

28. Ivi, 46.

29. Ivi, 47 .

30. JESI $2000,47$.

31. Ibidem.

32. Ivi, 49.

33. Cfr. KERÉNYI 1964 e JUNG, KERÉNYI 1972.

34. JESI, KERÉNYI 1999, 61.

35. CAVALLETTI, 2000, XV.

36. JESI 2000, 34.

37. Ivi, 50.

38. JESI, KERÉNYI 1999, 35.

39. Ivi, 51.

40. Ivi, 60 .

41. Ivi, 61 .

42. GNOLI 2000, 52 .

43. Ibidem.

44. CAVALLETTI 2001, 212. 
45. Benché Kierkegaard sia stato pubblicato per la prima volta nel 1972 è un testo degli anni sessanta a tutti gli effetti, poiché la sua gestazione era cominciata perlomeno dal 1959.

46. MANERA 2012, 48.

47. CAVAlletti, 2001, 203.

48. Ibidem.

49. CAVALLETTI 2001, 204.

50. BELLISARIO 1942, 9.

51. FERRARI, 4-5.

52. CAVALLETTI 2001, 205.

53. Ivi, 206-207.

54. CAVALLETTI 1999, 138

55. Ivi, 206

56. CAVALLETTI 2001, 207.

57. Ibidem.

58. BELPOLITI, MANERA 2010, 14.

59. JESI, KERÉNYI 1999, 114.

60. JESI 2001, 54.

61. Ivi, 55.

\section{RIASSUNTI}

La critica concorda sul rapporto stretto, in Furio Jesi, tra opera teorica e autobiografia. Le lettere che Furio Jesi scambia con il maestro Károly Kerényi dal 1964 al 1968 sono da considerarsi parte attiva del suo corpus teorico. La crisi tra allievo e maestro può essere letta in un confronto tra l'epistolario Jesi-Kerényi e i testi della fine degli anni settanta. Si tratta di specificare quale sia la figura di maestro che Jesi attribuisce a Kerényi e che poi sceglierà di incarnare. Le lettere del 1965 e del 1967 costituiscono, in particolare, uno spunto di riflessione su tale questione.

Furio Jesi and Károly Kerényi wrote letters to each other from 1964 to 1968 . The biographical value of those writings is indisputable, as they cast light on Jesi's years of apprenticeship. This article, on the other hand, aims to consider the relationship between the two scholars as an active part of Jesi's theoretical work. The key philosophical points that emerged from that exchange of letters, with a particular regard to the letters of 1965 and 1967, cast a new light on the role of the "disappeared master" that Jesi later on decides to embody.

\section{INDICE}

Keywords : Jesi Furio, Kerényi Károly, letters, Kierkegaard, maieutic

Parole chiave : Jesi Furio, Kerényi Károly, epistolario, Kierkegaard, maieutica 
AUTORE

PIERO ROSSO

Université de la Sorbonne Nouvelle (Paris 3), 1/5 rue Censier 75005, Paris

pieroross88(at)hotmail.com 